\title{
Diabetes and dementia - the two faces of Janus
}

\author{
Athanasia K. Papazafiropoulou ${ }^{1}$, Chris Koros², Andreas Melidonis ${ }^{3}$, Stavros Antonopoulos ${ }^{1}$
}

\author{
${ }^{1} 1^{\text {st }}$ Department of Internal Medicine and Diabetes Centre, Tzaneio General Hospital, \\ Piraeus, Greece \\ ${ }^{2} 1^{\text {st }}$ Department of Neurology, National and Kapodistrian University of Athens Medical \\ School, Eginition Hospital, Athens, Greece \\ ${ }^{3}$ Diabetes and Cardiometabolic Centre, Metropolitan Hospital, Piraeus, Greece
}

Submitted: 5 February 2020

Accepted: 18 April 2020

Arch Med Sci Atheroscler Dis 2020; 5: e186-e197

DOI: https://doi.org/10.5114/amsad.2020.97433

Copyright (c) 2020 Termedia \& Banach

\begin{abstract}
Patients with type 2 diabetes are at high risk for cognitive decline and dementia. Despite the limited data on the possible pathogenetic mechanisms, evidence suggests that cognitive decline, and thus dementia and Alzheimer's disease, might arise from a complex interplay between type 2 diabetes and the aging brain, including decreased insulin signalling and glucose metabolism, mitochondrial dysfunction, neuroinflammation, and vascular disease. Furthermore, there is increasing interest on the effects of antidiabetic agents on cognitive decline. There are many studies showing that antidiabetic agents might have beneficial effects on the brain, mainly through inhibition of oxidative stress, inflammation, and apoptosis. In addition, experimental studies on patients with diabetes and Alzheimer's disease have shown beneficial effects on synaptic plasticity, metabolism of amyloid- $\beta$, and microtubule-associated protein tau. Therefore, in the present review, we discuss the effects of antidiabetic agents in relation to cognitive decline, and in particular dementia and Alzheimer's disease, in patients with type 2 diabetes.
\end{abstract}

Key words: glucagon-like peptide-1 receptor agonists, sodium-glucose cotransporter- 2 inhibitors, dementia, Alzheimer's disease, neurodegenerative diseases, type 2 diabetes.

\section{Introduction}

It is well established that the prevalence of type 2 diabetes (T2D) is increasing worldwide, with more than 380 million people currently being diabetic [1]. Diabetic complications, peripheral and autonomic neuropathy, retinopathy, cerebrovascular and cardiovascular disease, and chronic kidney disease act negatively on quality of life and most of all increase the morbidity and mortality of patients with $\operatorname{T2D}[2,3]$. Cognitive decline has been recognised as another diabetic complication, resulting in mild cognitive impairment $(\mathrm{MCl})$ or dementia [4]. Furthermore, both epidemiological and experimental studies have demonstrated a link between Alzheimer's disease (AD) and T2D. Most of the studies emphasise the pathogenetic role of insulin resistance (IR) in impairing neuronal function, and some authors refer to AD as "type 3 diabetes" [5]. However, there is evidence that the link between $A D$ and T2D includes decreased brain insulin signalling and glucose metabolism, mitochondrial dysfunction, neuroinflammation, neurotransmitter alteration, and vascular disease.

\author{
Corresponding author: \\ Athanasia K. \\ Papazafiropoulou \\ MD, MSc, PhD \\ $1^{\text {st }}$ Department \\ of Internal Medicine \\ and Diabetes Center \\ Tzaneio General Hospital \\ 1 Zanni and Afentouli St \\ Piraeus 18536, Greece \\ Phone: +30 697996483 \\ E-mail: \\ pathan@ath.forthnet.gr
}


$I R$ and raising the levels of endogenous insulin secretion are the main mechanisms for the antidiabetic treatment, and many classes of antidiabetic agents have been available for decades targeting the above two pathogenetic mechanisms; these include metformin, sulfonylurea, thiazolidinediones, and insulin. Recently, incretin-based therapies and sodium-glucose co-transporter-2 inhibitors (SGLT-2i) have become available for the treatment of T2D acting in different pathogenetic ways. However, all the above antidiabetic agents are of great interest in relation to prevent cognitive decline, and thus dementia and Alzheimer's disease because it has been shown to have pleiotropic beneficial effects on the brain, mainly through inhibition of oxidative stress, inflammation, apoptosis, synaptic plasticity, and metabolism of amyloid- $\beta(A \beta)$ and microtubule-associated protein tau [2-5].

Therefore, in the present review, we will discuss the potential of incretin-based therapies in relation to cognitive decline and dementia, in particular $A D$, in patients with $T 2 D$.

\section{Dementia and type 2 diabetes}

It is well established that T2D is implicated in cognitive deterioration. In fact, it represents a major risk factor for dementia. The prevalence of dementia related to diabetes has not been assessed in depth yet. Diabetic patients are more prone to develop dementia than non-diabetic individuals [6]. The incidence of dementia in a prospective analysis was higher in diabetic patients (14.9\%). The presence of Apolipoprotein E4 (ApoE4) further increased the incidence of cognitive problems. Both diabetes and $A D$ are age-dependent conditions. Given the expectations for future growth of elderly populations, both these disorders are expected to affect millions of patients.

Epidemiological data have shown the association between AD and T2D. A study of the large Danish National Patient Cohort demonstrated that in patients $>50$ years old there is a small diabetes-related increase in AD [7]. In a Taiwan registry study diabetes type 2 was linked to a $60 \%$ increase in $A D$ risk [8]. A number of smaller studies have also shown an increase in AD risk in diabetic populations.

Studies in humans have shown that T2D is associated with dementia. A meta-analysis [9] showed a $60 \%$ greater dementia risk in diabetic patients, with women exhibiting a more robust effect. The risk accounts for vascular dementia while it is lower for non-vascular dementia. Concerning $\mathrm{MCl}$ there is an $20 \%$ rise in diabetic patients. Even for demented individuals, diabetes is linked to lower performance in cognitive functions like episodic memory, verbal fluency, processing speed, and at- tention [10, 11]. Structural alterations were also evident including higher brain atrophy and lower striatal volume in diabetic patients [12]. Notably, persons with high fasting blood glucose exhibited more pronounced cognitive decline, and the risk for dementia was greater. In a Japanese cohort, higher glycose level correlated with a higher vascular dementia risk [13]. In a large longitudinal cohort, high fasting blood glucose was associated with greater impact on verbal and spatial abilities and on perceptual speed [14].

Cognitive decline is also reflected in macroscopic brain changes like global cerebral and white matter atrophy in T2D [15]. A meta-analysis showed $2 \%$ lower brain volumes in diabetic patients [16]. Data on the annual rate of atrophy in diabetic patients are still missing. The duration of diabetes has been linked to grey matter atrophy ( $1 \%$ decrease for every additional 10 years). White matter atrophy and infarct volume was also associated with the disease duration $[17,18]$.

\section{Endogenous and environmental factors contributing to dementia in diabetic patients}

The factors that predispose to T2D and dementia tend to overlap. These include endogenous factors such as oxidative damage, inflammation, or mitochondrial dysfunction and environmental factors like diet, physical activity, and exposure to toxins. However, hyperglycaemia is considered to be a keystone in the development of dementia in diabetic patients [19]. Glucotoxicity exerts damage to a number of cell populations mainly via oxidative stress and mitochondrial dysregulation. These conditions lead to brain IR and subsequently to accumulation of $A \beta$. Notably, glucose transporters (GLUT) 1 and 3 and even 4, which is insulin dependent, play a major role in brain glucose metabolism. Knockout animal models for the latter exhibit abnormal response to hyperglycaemia in brain areas such as the hypothalamic paraventricular nucleus [20].

Chronic rises in blood glucose result in chronic hyperinsulinaemia, which in turn causes chronic brain IR. This condition leads to distorted brain insulin signalling and finally eliminates insulin availability in the brain (by means of insulin transport reduction across the blood-brain barrier). Because insulin receptor is abundant in the hippocampus, insulin resistance and limited brain insulin signalling has an impact on cognitive functions [21]. Additionally, both insulin and leptin regulate synaptic function in different brain areas and may even have a protective role against neurodegeneration. Synaptic dysregulation is considered to be important in the pathogenesis of dementia in diabetes.

The hallmark of the most frequent form of dementia, $A D$, is the presence of amyloid-rich senile 
plaques and neurofibrillary tangles (tau protein). The $A \beta$ is produced by means of cleavage of the original protein (Amyloid precursor protein APP) by beta and gamma secretases. On the other hand, tau protein, especially in phosphorylated form, aggregates and forms neurofibrillary tangles [22]. In animal models of T2D, a high-fat diet can enhance $A \beta$ production and increase tau protein phosphorylation [23]. Animals fed a high sucrose diet exhibit IR, hyperinsulinaemia, and deposition of amyloid in their brains. This histological find is accompanied by cognitive deficits [24].

The molecular mechanism behind these effects is probably the inhibition of APP degradation and subsequent amyloid accumulation due to hyperglycaemia and brain IR. Tau phosphorylation is also increased. In streptozotocin diabetic mice these pathological features are accompanied by hippocampal atrophy, synaptic dysregulation, and poor performance in learning tests. In all of these models diabetic-related neuronal abnormalities lead to structural and functional alterations, which account for profound cognitive deficits [25].

Although the uptake of glucose by neurons does not depend on insulin action, neuronal cells express such receptors along with insulin growth factor type I receptors (IGF-1) [26, 27]. It has been proposed that fluctuations of glucose level, as is the case in diabetes, may facilitate cognitive deterioration. Control of sudden glucose rises (peaks) might eliminate the damaging effect in memory function of diabetic individuals [28]. Medication aiming to decrease glucose fluctuations can have neuroprotective effects. Glucose lowering medication like metformin decrease tau hyperphosphorylation by means of enhancing protein phosphatase 2A. Moreover, thiazolidinediones, like pioglitazone, are agonists of peroxisome proliferator-activated receptor $\gamma$ (PPAR- $\gamma$ ) and appear to act beneficially on cognitive [29].

T2D has been correlated with an increase in blood cholesterol and triglycerides as a part of metabolic syndrome. Dyslipidaemia has also been implicated in cognitive deficits. The connection of this condition to memory is exemplified by the role of cholesterol in the activity of secretases. By means of this action $A \beta$ protein production and aggregation is enhanced. Dyslipidaemia could thus influence cognitive functions in diabetic patients via the amyloid cleavage pathway [30].

In diabetes, an increased inflammation background might explain in part the triggering effects on brain dysfunction. Advanced glycosylation end products (AGEs) lead to inflammatory responses, which in turn play a role in blood-brain barrier integrity [31]. Subsequently, the disrupted barrier makes the brain parenchyma more vulnerable to toxic circulating products including fibrin, plasmin, thrombin, and other proteins. Normal neuron homeostasis is then severely disturbed. Furthermore, inflammatory mediators like interleukins, tumour necrosis factor $\alpha$, and C-reactive protein, which are abundant owing to diabetes-driven inflammation, have been implicated in cognitive deficits [32, 33].

Hypertension, which is common in diabetic patients as part of the metabolic syndrome, plays a role in the development of cerebrovascular complications of diabetes. The exact pathway remains obscure, but it has been shown that lowering blood pressure exerts a neuroprotective role in diabetic patients and decreases the possibility of dementia [34].

Vascular problems represent a common outcome of chronic diabetes either in the form of macrovascular or microvascular disease. The presence of cerebral infarcts that accumulate in the brain white matter have a severe impact on memory and other cognitive functions. This condition often results in mild cognitive impairment or even frank dementia (vascular dementia) [35]. Microvascular abnormalities like those observed in the retina are also associated with cognitive deterioration. The effect of diabetes on the retina effectively reflects the status in cerebral small vessels. The pathology background of this condition includes endothelium alterations and capillary vessel loss [36]. It has been demonstrated that the dysregulation of endothelium stems from toxic agents that are produced in diabetic patients, such as lipids and AGEs. The latter lead to the generation of reactive oxygen species (ROS), which in turn influence endothelial cells [37]. Moreover, ROS have many additional harmful properties including the activation of metalloproteinases and the decrease of vasodilatory substances. Overall, this condition impacts vascular permeability in the brain, and this represents an obstacle to neuronal homeostasis. The combination of macroand microvascular brain disease trigger cognitive decline in diabetic patients $[26,38]$.

Other factors could also contribute to the development of diabetes-induced cognitive decline. Calcium metabolism alterations also play a role in neurodegeneration in diabetic patients. The major mechanism is considered to be $\mathrm{Ca} /$ calmodulin-dependent protein kinase II modification in diabetes [39]. Amylin represents a hormone secreted by pancreatic beta cells. Increased amylin in diabetes or prediabetes results in oligomerisation and secretion of oligomers in plasma. Circulating oligomers influence brain parenchyma by causing amylin amyloid deposition, microbleeds, and subsequent inflammation and degeneration. These deposits have been traced in the brain parenchyma of patients with $\operatorname{AD}[40,41]$.

Additionally, the formation and accumulation of misfolded proteins in T2D leads to brain dam- 
age. The ability of neurons to cleave misfolded proteins is impaired. Furthermore, cerebrovascular disease further prohibits the clearance of misfolded protein and accelerates neuronal damage [42]. Neurogenesis is an important procedure of neural cell proliferation, but in the adult brain it is restricted mainly to the hippocampus. In diabetes, high blood glycose is associated with low trophic factor levels like brain-derived neurotrophic factor and impaired neurogenesis, which may have an indirect effect on cognitive functions in the long run [43].

The glymphatic brain system represents the glial component of the outer boundary of the perivascular space, which plays a crucial role in the clearance and turnover of substances between the brain parenchyma and the vessels. This system is severely affected in degenerative disease like $A D$ [44]. The main mechanism of glymphatic system dysfunction in $A D$ is the lack of efficiency in $A \beta$ clearance because toxic amyloid can be transported via low-density lipoprotein-related receptor I [45]. In AD, previous studies have demonstrated a defect in the clearance of amyloid via the glymphatic system. Furthermore, decreased aquaporin receptor expression in $A D$ has been linked to reduce $A \beta$ and tau clearance via the glymphatic system. Accordingly, in T2D, recent reports have shown decreased activity of the glymphatic system. IR and hyperglycaemia possibly play a role in this deficit [46]. Moreover, diabetic patients exhibit problems in the blood brain barrier permeability, a decline in norepinephrine secretion in the brain, and decreased ApoE levels. These conditions influence the function of the glymphatic system, and the resulting brain dysfunction leads to cognitive deterioration [47]. In diabetic patients an increased glymphatic influx of molecules to the cerebrospinal fluid is noted along with a reduction of clearance of parenchymal molecules, including amyloid [48]. Moreover, small-vessel disease is accompanied by glymphatic malfunction in diabetes. The blood-brain barrier when intact is protective against a number of neurotoxins that could affect normal brain activity. On the other hand, sleep has been linked to amelioration of the impaired function of the glymphatic system in diabetic patients and to decrease the impact of diabetes on cognitive functions of affected individuals [44, 49].

A number of environmental factors have been implicated in the pathogenesis of both T2D and AD. Air pollution has been linked to T2D. Large longitudinal studies have demonstrated an increased risk of diabetes in individuals exposed to environmental toxins. Air pollution is also related to the development of $A D$, with higher exposures doubling the risk for AD. Currently, few studies have assessed the possible interaction of air pollution and T2D on cognition. The results are indicative of the influence of metabolic disturbances triggered by toxic substances on $A D$ risk. A number of other environmental toxic substances like pesticides used in agriculture have also been implicated in the development of both T2D and dementia [1].

\section{Antidiabetic agents and dementia}

\section{Metformin}

Metformin is a biguanide, and for than more half a century it has remained the first-line therapy for T2D. Its action occurs by the inhibition of gluconeogenesis in the liver and activation of the liver kinase B1 (LKB1)/AMPK pathway through the inhibition of the mitochondrial respiratory-chain complex [50]. The existing literature data show that metformin therapy in T2D patients is associated with a significantly lower risk of dementia and cognitive impairment. Metformin's neuroprotective effects are explained in part by the activation of AMPK-dependent pathway [51] and its effect on IR $[52,53]$. The later was confirmed by Lin et al., showing that metformin can improve cognitive function in patients with non-dementia vascular cognitive impairment and abnormal glucose metabolism by a beneficial result on IR [54].

It is known that $A D$ is characterised by deposition of $A \beta$ plaques, neurofibrillary tangles, and neuronal loss, accompanied by neuroinflammation. A study in APP/PS1 mice showed that metformin attenuated spatial memory deficit and neuron loss, and decreased $A \beta$ plaque load and chronic inflammation in the hippocampus and cortex [55]. In addition, in an experimental model in male Wistar rats, metformin offered a protective effect against scopolamine-induced cognitive impairment, mainly due to a significant reduction of inflammation and to a lesser extent to reduction of oxidative stress [56].

A large retrospective study in 5528 elderly patients with T2D, with a median follow-up of 5.2 years, showed that long-term metformin therapy was associated with lower incidence of neurodegenerative disease [57]. In accordance, a retrospective study of 17,200 new users of metformin and 11,440 new users of sulfonylureas showed that the hazard ratio (HR) for dementia in metformin vs. a sulfonylurea group was 0.67 (95\% confidence interval $(95 \% \mathrm{Cl}): 0.61-0.73)$ and 0.78 (95\% Cl: $0.72-0.83$ ) for those $<75$ and $\geq 75$ years of age, respectively [58]. Another study, from 2000 to 2015, in 73,761 African American and white patients, showed that metformin was associated with a substantially lower risk of dementia compared to sulfonylurea initiation among African American patients aged 50 to 64 years $(H R=0.6$; 95\% Cl: 0.45-0.81) [59]. 
The population-based Singapore Longitudinal Aging Study in older patients with T2D showed that metformin use was associated with a low risk of cognitive impairment [60]. The favourable effect of metformin therapy on risk of dementia $(\mathrm{HR}=0.76,95 \% \mathrm{Cl}: 0.58-0.98)$ was also confirmed in a study using data from 800,000 patients in Taiwan's National Health Insurance database [61]. Even in the presence of mild cognitive impairment or mild dementia due to AD metformin was associated with improvement in learning/memory and attention [62]. However, a cohort study in 4651 patients using Taiwan's National Health Insurance Research Database, with a 12-year follow-up, showed that patients on metformin therapy had a higher risk of Parkinson's disease (PD) $(\mathrm{HR}=2.27,95 \% \mathrm{Cl}: 1.68-3.07)$, and an increased risk of all-cause dementia $(\mathrm{HR}=1.66,95 \% \mathrm{Cl}$ : $1.35-2.04), A D(H R=2.13,95 \% \mathrm{Cl}: 1.20-3.79)$ and vascular dementia ( $\mathrm{HR}=2.30,95 \% \mathrm{Cl}: 1.25-4.22)$ compared to the non-metformin group [63].

On the basis of the presented results, it seems that metformin exerts beneficial effects regarding dementia and AD. However, the exact pathogenetic mechanism is not yet fully known. Therefore, further well-designed, multicentre, placebo-controlled, randomised clinical studies are necessary in order to explain the beneficial effects of metformin on dementia and AD.

\section{Sulphonylurea}

The existing literature data regarding the effect of sulphonylurea on dementia are very limited. It has been shown that glimepiride might reduce synapse damage and hence delay the progression of cognitive decline in $A D$ [64]. It seems that the protective effects of glimepiride were multi-faceted: altered synaptic membranes, increased synaptic gangliosides, and altered cell signalling [64]. However, further studies using sulphonylurea are needed in order to explore the potential effect that might have on dementia as well as the effect of hypoglycaemic episodes that are fully related with sulphonylurea therapy on the risk of dementia.

\section{Glitazones}

PPAR- $\gamma$ agonists are insulin sensitising drugs indicated mostly for T2D patients with IR. Many mechanisms have been proposed in order to explain the beneficial effects of PPAR- $\gamma$ agonists on AD [65]. In AD models, PPAR- $\gamma$ agonists improved learning and reduced amyloid burden and inflammation [66]. An experimental model with diabetes-induced vascular dementia showed that treatment with PPAR- $\gamma$ agonists, pioglitazone, and rosiglitazone significantly reversed diabetes-in- duced impairment of learning and memory behaviour, and endothelial function [67].

Several studies have shown that pioglitazone treatment improved cognitive performance, lowered oxidative stress, and improved cerebral glucose utilisation [68-70]. In accordance, a study using aged APP transgenic mice with severe cerebrovascular and memory deficits demonstrated that pioglitazone therapy not only overcame cerebrovascular dysfunction and altered neurometabolic coupling, but also counteracted cerebral oxidative stress, glial activation, and cholinergic denervation [71]. Furthermore, in another model, combination treatment with leptin and pioglitazone reduced plaque-associated neuritic pathology and synapse loss, as well as neocortical glial response [72].

Another study in a mouse model of AD with accelerated $A \beta$ deposition and tau pathology showed that after 4 months of pioglitazone treatment, animals showed improved learning on the active avoidance task, reduced serum cholesterol, decreased hippocampal $A \beta$ and tau deposits, and enhanced short- and long-term plasticity [73]. It has also been shown that low-dose pioglitazone might increase the expression of low-density lipoprotein receptor-related protein 1 (LRP1), which upregulates the clearance of $A \beta$, using human brain microvascular endothelial cells [74]. Another study found increased phosphorylation levels of CRMP2 as well as increased p35 protein levels in the cerebellum of APP/PS1 mice. Pioglitazone normalised the p35 protein and CRMP2 phosphorylation levels in the cerebellum. Interestingly, impaired motor coordination ability and long-term depression in APP/PS1 mice were ameliorated by pioglitazone treatment at the pre-A $\beta$ accumulation stage [75]. However, a study failed to show any beneficial effects of pioglitazone therapy and showed that chronic treatment with pioglitazone decreased cerebral glucose utilisation in vivo. This evidence does not support the hypothesis that pioglitazone could act as a metabolic enhancer in $A D$, and it raises the question of how thiazolidinediones could be beneficial in neurodegenerative diseases [76].

A study using data from 5048 patients with new-onset T2D from the Taiwan National Health Insurance Research Database, during 1999-2006, showed that rosiglitazone treatment had a neutral effect on the risk of dementia $(\mathrm{HR}=0.86,95 \% \mathrm{Cl}$ : 0.70-1.15) [77]. However, another study from 2004 to 2009, using again data from the Taiwan National Health Insurance Research Database, in 6401 patients with T2D, who were treated with pioglitazone and 12,802 age- and sex-matched patients with T2D, who were never treated with pioglitazone and who were free of dementia at 
baseline, showed that the risk of dementia decreased by $23 \%$ in the pioglitazone-treated cohort compared to the control group after adjustment for age, sex, hypertension, and stroke ( $H R=0.77$, 95\% Cl: 0.62-0.96) [78]. The same finding was confirmed when pioglitazone was used in combination with metformin in the same database [79]. It is noteworthy that a lower incidence of dementia was found in users of metformin plus pioglitazone compared with users of metformin plus rosiglitazone [79], showing a possible different action of the two agents on dementia. Another observational study, from 2004-2010, using data from 145,928 subjects aged $\geq 60$ years, who were free of dementia at baseline, showed that longterm use of pioglitazone reduced the risk of dementia by $47 \%$ [80].

Therefore, there is insufficient evidence to support any beneficial effect of rosiglitazone in AD patients. However, the efficacy of pioglitazone seems to be promising and needs to be further confirmed in well-designed trials with large sample sizes. The cardiovascular risk associated with this class of medications is also a concern and has limited their use to just selected patients with T2D.

\section{Incretins}

\section{Dipeptidyl peptidase 4 inhibitors}

There is sufficient evidence based on experimental and clinical trials to indicate that dipeptidyl peptidase 4 inhibitors (DPP-4i) have neuroprotective properties, and several mechanisms have been proposed in order to explain the neuroprotective properties of DPP-4i. An animal study showed that sitagliptin treatment improved working and reference memories with reduction in HOMA-IR and incensement of the hypothalamic acetylcholine level, possibly through increased AdipoR1 expression [81]. The impact of sitagliptin on the deposition of $A \beta$ within the brain was demonstrated in an animal model of $A D$, while in the same study sitagliptin improved deficits in memory-related behavioural paradigms [82]. Another study, using a model of AD mice, showed that sitagliptin reduced the escape latency times in the learning phase and elongated the time spent in the target quadrant. Furthermore, sitagliptin significantly reduced $A \beta$ plaque deposition and elevated the spine density and the protein levels of synaptoneurosome GluA1- and GluA2-containing AMPA receptor in the brain of an APP/PS1 mouse model [83]. Finally, a human study in AD patients showed that sitagliptin therapy was associated with an increase in the Mini-Mental State Examination scores, showing significant improvement of cognitive function in elderly T2D patients [84]. Unexpectedly, one study found that sitagliptin was not effective against pathological tau phosphorylation in the hippocampus of OLETF T2D rats. On the contrary, sitagliptin aggravated tau phosphorylation. This paradoxically increased tau phosphorylation was attributed to activation of the glycogen synthase kinase $3 \beta$ [85].

A study tested the in vivo actions of glucagon-like peptide-1 (GLP-1) in the diabetic brain by a 10-week treatment of ZDF rats with alogliptin treatment. Alogliptin increased the circulating levels of GLP-1 by $125 \%$ and decreased blood glucose in by $59 \%$. Results like the above suggest that incretin therapies might reduce cognitive decline in aging T2D patients and also might have a favourable effect in treating $A D$ [86]. Another DPP-4i, linagliptin, significantly protected against $A \beta$-induced cytotoxicity, and prevented the activation of glycogen synthase kinase $3 \beta$ and tau hyperphosphorylation by restoring insulin downstream signalling, suggesting a finding that might have a therapeutic impact in the reduction of $A \beta$-induced impairment of insulin signalling and neurotoxicity in $A D$ pathogenesis [87]. In another study, linagliptin improved brain incretin levels and attenuated $A \beta$, tau phosphorylation, and neuroinflammation [88].

A study investigated the role of vildagliptin in diabetes-induced vascular endothelial dysfunction and subsequent vascular dementia in rats. Administration of vildagliptin significantly attenuated impairment of learning, memory, endothelial function, and blood-brain barrier permeability [89]. Results of another two studies showed that vildagliptin had a protective effect against cognitive deficits by reducing tau phosphorylation and increasing the expression of proteins associated with synaptic plasticity in the hippocampus of the tested animal models $[90,91]$. Attenuation of $A \beta$, tau phosphorylation, and inflammatory markers and an improvement in hippocampal GLP-1 and memory retention were also observed following saxagliptin treatment [92].

Summarising, there is supporting evidence that DPP-4i might prevent cognitive decline. However, more clinical trials are needed in patients at high risk of cognitive decline, including those with and without diabetes, in order to establish a protective association.

\section{Glucagon-like peptide-1 receptor agonists}

The main described role in the brain for GLP-1 is in the regulation of food intake, because it is primarily distributed on hypothalamic nuclei [93]. GLP-1 receptor expression has also been demonstrated in the thalamus, brainstem, lateral septum, subfornical organ, and the area postrema, suggesting roles in memory, attention, and behaviour, all of which are affected in neurodegen- 
erative conditions such as AD [94, 95]. Exendin-4 (Ex-4), a GLP-1 receptor agonist, has been shown to protect neurons from diabetes-associated glucose metabolic dysregulation in vitro and might have therapeutic value in the treatment of T2D-related $A D$ [96]. A study using exenatide therapy showed that it had beneficial effects on impaired cognitive performance and hippocampal neuronal viability in $A D$ by suppressing the inflammation response and increasing cholinergic activity [97]. One possible mechanism for the neuroprotection of Ex-4 appears to be the prevention of the hyperphosphorylation of AD-associated tau protein due to increased insulin signalling pathway in the brain $[98,99]$. Furthermore, the existing data suggest that GLP-1 receptor agonists can protect neurons against metabolic memory via Forkhead box class $\mathrm{O}$ (FoxO) pathways, silent information regulator 2 homolog 1-dependent deacetylation, and protein kinase $\mathrm{B}$-dependent phosphorylation of FoxO1 [100].

Liraglutide can increase progenitor cell proliferation, and induce neuroblast differentiation and subsequent differentiation into neurons in an $A D$ mouse model [101]. Also, the neuroprotective effects of liraglutide on diabetes-induced cognitive impairments are associated with the improvements of hippocampal synapses and inhibition of neuronal apoptosis [102]. Moreover, liraglutide enhances and reverses the impairments of synaptic plasticity induced by $A \beta$ fragments, showing a significant effect on neurotransmission in the brain in AD [103]. Liraglutide treatment significantly decreases IR aberrations in conjunction with a concomitant decrease in amyloid plaque load. Liraglutide also induces a highly significant reduction in astrocytosis and microglial number associated with both plaques and IR pathology [104]. Subcutaneous administration of liraglutide prevented memory impairments and alleviated the ultra-structural changes of pyramidal neurons and chemical synapses in the hippocampal region. Furthermore, liraglutide reduced A $\beta 1-42$-induced tau phosphorylation via the protein kinase $B$ and glycogen synthase kinase- $3 \beta$ pathways [105]. Liraglutide restores both peripheral and brain insulin sensitivity and ameliorates tau hyperphosphorylation in rats with $\mathrm{T} 2 \mathrm{D}$, supporting the potential use of liraglutide for the prevention and treatment of $A D$ in individuals with T2D [106]. Another study using hippocampal neuronal cultures determined one of the possible mechanisms of neuroprotection by liraglutide, which involves activation of the protein kinase A signalling pathway [107].

Furthermore, liraglutide showed restorative effects in late-stage $A D$ in mice. Overall plaque load was reduced by $33 \%$, and inflammation was reduced by $30 \%$, while the neuronal progenitor cell count in the dentate gyrus was increased by $50 \%$ [108]. Liraglutide can also delay or partially halt the progressive decline in memory function associated with hippocampal neuronal loss in a mouse model of pathological aging with characteristics of neurobehavioural and neuropathological impairments observed in early-stage sporadic AD [109]. However, an experimental study showed that long-term liraglutide treatment exhibited no effect on cerebral plaque load in two transgenic mouse models of low- and high-grade amyloidosis, which suggests differential sensitivity to long-term liraglutide treatment in various transgenic mouse models mimicking distinct pathological hallmarks of AD [110]. Another recent study reported neural effects of liraglutide in a middle-aged population with subjective cognitive complaints [111].

Another GLP-1 receptor agonist, lixisenatide, has been shown to reduce amyloid plaques, neurofibrillary tangles, and neuroinflammation in an experimental model showing promising results [112]. Lixisenatide, by affecting the PI3K-AktGSK3 $\beta$ pathway, can prevent $A \beta$-related impairments in synaptic plasticity and spatial memory of rats, suggesting that lixisenatide may be a novel and effective treatment for AD [113].

Finally, novel dual GLP-1/gastric inhibitory peptide (GIP) receptor agonists have been developed to treat T2D, and they also show good neuroprotective effects that are superior to single GLP-1 analogues. Several newer dual analogues have been tested that have been engineered to cross the blood-brain barrier. They show clear neuroprotective effects by reducing inflammation and oxidative stress and apoptotic signalling and protecting memory formation, synaptic numbers and synaptic activity, motor activity, dopaminergic neurons, cortical activity, and energy utilisation in the brain [114]. A study using a novel dual GLP-1/ GIP receptor agonist (DA-JC4) once-daily for 14 days showed significant prevention of spatial learning and decreased phosphorylated tau levels in the rat cerebral cortex and hippocampus. DA-JC4 also alleviated the chronic inflammation response in the brain, as well as apoptosis [115]. Furthermore, a novel unimolecular GLP-1/GIP/ Gcg triagonist has been shown to be efficacious in ameliorating cognitive deficits and pathological damage of 3xTg-AD mice, suggesting that the triagonist might be beneficial in the treatment of $A D$ [116]. In another study, treatment with the novel triagonist reduced the total amount of $A \beta$, and reduced neuroinflammation (activated microglia and astrocytes) and oxidative stress in the cortex and hippocampus in rats. These findings show that novel triagonists are a promising lead for the design of future treatment strategies in AD [117]. 
The above findings support the potential use of GLP-1 receptor agonists for the prevention and treatment of $A D$ in individuals with T2D. Therefore, larger and longer duration studies are warranted to determine whether GLP-1 receptor agonists have neuroprotective benefits in individuals at risk for $A D$

\section{Sodium glucose Co-transporter 2 inhibitors}

There are very limited data regarding the potential effect of the newest antidiabetic treatment category, SGLT2i. A recent study showed that canagliflozin, a SGLT2i, might act as a potent inhibitor of acetylcholinesterase which is a primary target for AD therapy [118]. The same finding was observed using another SGLT2i, dapagliflozin. Thus, the above results might form the basis of future therapy against diabetes-associated neurological disorders [119]. However, large, well-designed trials are needed in order to find the impact of SGLT2i therapy on dementia and AD.

\section{Insulin}

Insulin modulates neurotransmitter release and synaptic plasticity, the basis for cognition, learning, and memory [119-121]. Animal models and human studies have extensively documented impaired insulin signalling and degradation in $A D$ and T2D. It is known that insulin promotes tau hypophosphorylation, which stabilises microtubules and promotes tubulin polymerisation. Therefore, excess exogenous insulin might play a role in overcoming the decreased utilisation and transport of glucose in patients with AD. In addition, it has been demonstrated that intranasal insulin therapy may have beneficial effects on cognition and function in patients with AD [122]. Insulin is a multipotent hormone regulating not only glucose levels, but also cell survival and synaptic plasticity mechanisms of neurons. Insulin has been shown to facilitate reduction of intracellular amyloid plaque and downregulation of $A \beta$-derived diffusible ligand-binding sites [123]. On the other hand, insulin-induced hypoglycaemia causes adaptive changes in the brain, including an improved ability to use alternative fuels [74].

A study compared the effects of insulin glargine U100, detemir, and degludec on neural functions in an animal model. The results of the study showed that insulin glargine U100 enhanced memory functions and suppressed depression-like behaviour. These effects were more potent than those of detemir [124]. Another study aimed to determine whether treatment with intranasal insulin detemir or regular insulin improves cognition and daily functioning in adults with $\mathrm{MCl}$ or $A D$, and it showed that the regular insulin-treated group had better memory compared with placebo.
Furthermore, regular insulin treatment was associated with reduction in the tau-P181/A $\beta 42$ ratio, while no significant effects were observed for the detemir-assigned group compared with the placebo group [125]. Another trial examined whether intranasal insulin detemir improves cognition or daily functioning in adults with $\mathrm{MCl}$ or $\mathrm{AD}$. The results revealed a treatment effect for verbal and visuospatial working memory for subjects who received the high dose of intranasal insulin detemir. However, no significant differences were found for daily or executive functioning [126].

\section{Conclusions}

A lot of the existing literature data confirm a relation between T2D and dementia and AD with possible pathogenetic links to decreased brain insulin signalling, mitochondrial dysfunction, neuroinflammation, alteration of neurotransmitters, and vascular disease. On the other hand, many studies have shown that antidiabetic agents, especially metformin, glitazones, and GLP-1 receptor agonists, have a potential therapeutic role in the prevention or treatment of dementia and AD. However, the underlying mechanisms between antidiabetic agents and neurodegenerative diseases are not fully understood, and further studies are needed in order to improve knowledge on the possible pathophysiological links between them and, most of all, on their therapeutic implications.

\section{Conflict of interest}

The authors declare no conflict of interest.

\section{References}

1. International Diabetes Federation. IDF Diabetes Atlas, $6^{\text {th }}$ edn. 2013.

2. Duarte Al, Moreira PI, Oliveira CR. Insulin in central nervous system: more than just a peripheral hormone. J Aging Res 2012; 2012: 384017.

3. Campbell RK. Clarifying the role of incretin-based therapies in the treatment of type 2 diabetes mellitus. Clin Ther 2011; 33: 511-27.

4. de la Monte SM, Wands JR. Alzheimer's disease is type 3 diabetes-evidence reviewed. J Diabetes Sci Technol 2008; 2: 1101-13.

5. Koekkoek PS, Kappelle LJ, van den Berg E, et al. Cognitive function in patients with diabetes mellitus: guidance for daily care. Lancet Neurol 2015; 14: 329-40.

6. Paul KC, Jerrett M, Ritz B. Type 2 diabetes mellitus and Alzheimer's disease: overlapping biologic mechanisms and environmental risk factors. Curr Environ Health Rep 2018; 5: 44-58.

7. Schmidt M, Schmidt SA, Sandegaard IL, et al. The Danish National Patient Registry: a review of content, data quality, and research potential. Clin Epidemiol 2015; 7: 449-90.

8. Wang KC, Woung LC, Tsai MT, et al. Risk of Alzheimer's disease in relation to diabetes: a population-based cohort study. Neuroepidemiology 2012; 38: 237-44. 
9. Gould CE, Beaudreau SA, Salman H. Diabetes is associated with cognitive impairment no dementia in the Aging, Demographics, and Memory Study (ADAMS). Int Psychogeriatr 2013; 25: 167-8.

10. Monette MC, Baird A, Jackson DL. A meta-analysis of cognitive functioning in nondemented adults with type 2 diabetes mellitus. Can J Diabetes 2014; 38: 401-8.

11. Palta P, Golden SH, Teresi J, et al. Mild cognitive dysfunction does not affect diabetes mellitus control in minority elderly adults. J Am Geriatr Soc 2014; 62: 2363-8.

12. Kumar R, Anstey KJ, Cherbuin N, et al. Association of type 2 diabetes with depression, brain atrophy, and reduced fine motor speed in a 60- to 64-year-old commu nity sample. Am J Geriatr Psychiatry 2008; 16: 989-98.

13. Ohara T, Doi Y, Ninomiya T, et al. Glucose tolerance status and risk of dementia in the community: the $\mathrm{Hi}$ sayama study. Neurology 2011; 77: 1126-34.

14. Seetharaman S, Andel R, McEvoy C, et al. Blood glucose, diet-based glycemic load and cognitive aging among dementia-free older adults. J Gerontol A Biol Sci Med Sci 2015; 70: 471-9.

15. van Harten B, Oosterman JM, Potter van Loon BJ, et al. Brain lesions on MRI in elderly patients with type 2 diabetes mellitus. Eur Neurol 2007; 57: 70-4.

16. Zhang T, Shaw M, Humphries J, et al. Higher fasting plasma glucose is associated with striatal and hippocampal shape differences: the 2 sweet project. BMJ Open Diabetes Res Care 2016; 4: e000175.

17. Tiehuis AM, van der Graaf Y, Visseren FL, et al.; SMART Study Group. Diabetes increases atrophy and vascular lesions on brain MRI in patients with symptomatic arterial disease. Stroke 2008; 39: 1600-3.

18. Cherbuin N, Walsh El. Sugar in mind: Untangling a sweet and sour relationship beyond type 2 diabetes. Front Neuroendocrinol 2019; 54: 100769.

19. Lee HJ, Seo HI, Cha HY, et al. Diabetes and Alzheimer's disease: mechanisms and nutritional aspects. Clin Nutr Res 2018; 7: 229-40.

20. Vannucci SJ, Koehler-Stec EM, Li K, et al. GLUT4 glucose transporter expression in rodent brain: effect of diabetes. Brain Res 1998; 797: 1-11.

21. Neth BJ, Craft S. Insulin resistance and Alzheimer's disease: bioenergetic linkages. Front Aging Neurosci 2017; 9: 345.

22. Armstrong RA. The molecular biology of senile plaques and neurofibrillary tangles in Alzheimer's disease. Folia Neuropathol 2009; 47: 289-99.

23. Devi L, Alldred MJ, Ginsberg SD, et al. Mechanisms underlying insulin deficiency-induced acceleration of beta-amyloidosis in a mouse model of Alzheimer's disease. PLoS One 2012; 7: e32792.

24. Cao D, Lu H, Lewis TL, Li L. Intake of sucrose-sweetened water induces insulin resistance and exacerbates memory deficits and amyloidosis in a transgenic mouse model of Alzheimer disease. J Biol Chem 2007; 282: 36275-82

25. Wang JQ, Yin J, Song YF, et al. Brain aging and AD-like pathology in streptozotocin-induced diabetic rats. J Diabetes Res 2014; 2014: 796840.

26. Verma N, Despa F. Contributing factors to diabetic brain injury and cognitive decline. Diabetes Metab J 2019; 43: 560-7.

27. Fernandez AM, Torres-Alemán I. The many faces of insulin-like peptide signalling in the brain. Nat Rev Neurosci 2012; 13: 225-39.
28. Rawlings AM, Sharrett AR, Mosley TH, et al. Glucose peaks and the risk of dementia and 20-year cognitive decline. Diabetes Care 2017; 40: 879-86.

29. Gault VA, Hölscher C. GLP-1 receptor agonists show neuroprotective effects in animal models of diabetes. Peptides 2018; 100: 101-7.

30. Perlmuter LC, Nathan DM, Goldfinger SH, et al. Triglyceride levels affect cognitive function in noninsulindependent diabetics. J Diabet Complications 1988; 2 210-3.

31. Viswanathan A, Rocca WA, Tzourio C. Vascular risk factors and dementia: how to move forward? Neurology 2009; 72: 368-74.

32. Prasad S, Sajja RK, Naik P, et al. Diabetes mellitus and blood-brain barrier dysfunction: an overview. J Pharmacovigil 2014; 2: 125.

33. Gorska-Ciebiada M, Saryusz-Wolska M, Borkowska A et al. C-reactive protein, advanced glycation end products, and their receptor in type 2 diabetic, elderly patients with mild cognitive impairment. Front Aging Neurosci 2015; 7: 209

34. Hassing LB, Hofer SM, Nilsson SE, et al. Comorbid type 2 diabetes mellitus and hypertension exacerbates cognitive decline: evidence from a longitudinal study. Age Ageing 2004; 33: 355-61.

35. Biessels GJ, Strachan MW, Visseren FL, et al. Dementia and cognitive decline in type 2 diabetes and prediabetic stages: towards targeted interventions. Lancet Diabetes Endocrinol 2014; 2: 246-55.

36. Ding J, Patton N, Deary IJ, et al. Retinal microvascular abnormalities and cognitive dysfunction: a systematic review. Br J Ophthalmol 2008; 92: 1017-25.

37. Basta G, Schmidt AM, De Caterina R. Advanced glycation end products and vascular inflammation: im plications for accelerated atherosclerosis in diabetes. Cardiovasc Res 2004; 63: 582-92.

38. Quaegebeur A, Lange C, Carmeliet P. The neurovas cular link in health and disease: molecular mechanisms and therapeutic implications. Neuron 2011; 71: 406-24.

39. Erickson JR, Pereira L, Wang L, et al. Diabetic hyperglycaemia activates CaMKII and arrhythmias by O-linked glycosylation. Nature 2013; 502: 372-6.

40. Jackson K, Barisone GA, Diaz E, et al. Amylin deposition in the brain: a second amyloid in Alzheimer disease? Ann Neurol 2013; 74: 517-26.

41. Fawver JN, Ghiwot Y, Koola C, et al. Islet amyloid polypeptide (IAPP): a second amyloid in Alzheimer's disease. Curr Alzheimer Res 2014; 11: 928-40.

42. Grimmer T, Faust M, Auer F, et al. White matter hyperintensities predict amyloid increase in Alzheimer's disease. Neurobiol Aging 2012; 33: 2766-73.

43. Krabbe KS, Nielsen AR, Krogh-Madsen R, et al. Brain-derived neurotrophic factor (BDNF) and type 2 diabetes. Diabetologia 2007; 50: 431-8.

44. Kim YK, Nam KI, Song J. The glymphatic system in diabetes-induced dementia. Front Neurol 2018; 9: 867.

45. Zuroff L, Daley D, Black KL, et al. Clearance of cerebral Abeta in Alzheimer's disease: reassessing the role of microglia and monocytes. Cell Mol Life Sci 2017; 74: 2167-201.

46. Zeppenfeld DM, Simon M, Haswell JD, et al. Association of perivascular localization of aquaporin-4 with cognition and Alzheimer disease in aging brains. JAMA Neurol 2017; 74: 91-9.

47. Peila R, Rodriguez BL, Launer LJ; Honolulu-Asia Aging Study. Type 2 diabetes, APOE gene, and the risk for 
dementia and related pathologies: the Honolulu-Asia Aging Study. Diabetes 2002; 51: 1256-62.

48. Luchsinger JA, Gustafson DR. Adiposity, type 2 diabetes, and Alzheimer's disease. J Alzheimers Dis 2009; 16: 693-704.

49. Longordo F, Kopp C, Lüthi A. Consequences of sleep deprivation on neurotransmitter receptor expression and function. Eur J Neurosci 2009; 29: 1810-9.

50. Rena G, Pearson ER, Sakamoto K. Molecular mechanism of action of metformin: old or new insights? Diabetologia 2013; 56: 1898-906.

51. Markowicz-Piasecka M, Sikora J, Szydłowska A, et al. Metformin - a future therapy for neurodegenerative diseases. Pharm Res 2017; 34: 2614-27.

52. Chen Y, Zhang J, Zhang B, et al. Targeting insulin signaling for the treatment of Alzheimer's disease. Curr Top Med Chem 2016; 16: 485-92.

53. Viollet B, Guigas B, Sanz Garcia N, et al. Cellular and molecular mechanisms of metformin: an overview. Clin Sci (Lond) 2012; 122: 253-70.

54. Lin Y, Wang K, Ma C, et al. Evaluation of metformin on cognitive improvement in patients with non-dementia vascular cognitive impairment and abnormal glucose metabolism. Front Aging Neurosci 2018; 10: 227.

55. Ou Z, Kong X, Sun X, et al. Metformin treatment prevents amyloid plaque deposition and memory impairment in APP/PS1 mice. Brain Behav Immun 2018; 69: 351-63.

56. Mostafa DK, Ismail CA, Ghareeb DA. Differential metformin dose-dependent effects on cognition in rats: role of Akt. Psychopharmacology (Berl) 2016; 233: 2513-224.

57. Shi Q, Liu S, Fonseca VA, et al. Effect of metformin on neurodegenerative disease among elderly adult US veterans with type 2 diabetes mellitus. BMJ Open 2019; 9: e024954.

58. Orkaby AR, Cho K, Cormack J, et al. Metformin vs sulfonylurea use and risk of dementia in US veterans aged $\geq 65$ years with diabetes. Neurology 2017; 89: 1877-85.

59. Scherrer JF, Morley JE, Salas J, et al. Association between metformin initiation and incident dementia among African American and white veterans health administration patients. Ann Fam Med 2019; 17: 352-62.

60. Ng TP, Feng L, Yap KB, et al. Long-term metformin usage and cognitive function among older adults with diabetes. J Alzheimers Dis 2014; 41: 61-8.

61. Hsu CC, Wahlqvist ML, Lee MS, et al. Incidence of dementia is increased in type 2 diabetes and reduced by the use of sulfonylureas and metformin. J Alzheimers Dis 2011; 24: 485-93.

62. Koenig AM, Mechanic-Hamilton D, Xie SX, et al. Effects of the insulin sensitizer metformin in Alzheimer disease: pilot data from a randomized placebo-controlled crossover study. Alzheimer Dis Assoc Disord 2017; 31: 107-13.

63. Kuan YC, Huang KW, Lin CL, et al. Effects of metformin exposure on neurodegenerative diseases in elderly patients with type 2 diabetes mellitus. Prog Neuropsychopharmacol Biol Psychiatry 2017; 79: 77-83.

64. Osborne C, West E, Nolan W, et al. Glimepiride protects neurons against amyloid-beta-induced synapse damage. Neuropharmacology 2016; 101: 225-36.

65. van Bilsen M, van Nieuwenhoven FA. PPARs as therapeutic targets in cardiovascular disease. Expert Opin Ther Targets 2010; 14: 1029-45.
66. Jiang $\mathrm{Q}$, Heneka $M$, Landreth GE. The role of peroxisome proliferator-activated receptor-gamma (PPARgamma) in Alzheimer's disease: therapeutic implications. CNS Drugs 2008; 22: 1-14.

67. Sharma B, Singh N. Behavioral and biochemical investigations to explore pharmacological potential of PPAR-gamma agonists in vascular dementia of diabetic rats. Pharmacol Biochem Behav 2011; 100: 320-9.

68. Pathan AR, Viswanad B, Sonkusare SK, et al. Chronic administration of pioglitazone attenuates intracerebroventricular streptozotocin induced-memory impairment in rats. Life Sci 2006; 79: 2209-16.

69. Kaur B, Singh N, Jaggi AS. Exploring mechanism of pioglitazone-induced memory restorative effect in experimental dementia. Fundam Clin Pharmacol 2009; 23: 557-66.

70. Gupta R, Gupta LK. Improvement in long term and visuo-spatial memory following chronic pioglitazone in mouse model of Alzheimer's disease. Pharmacol Biochem Behav 2012; 102: 184-90.

71. Nicolakakis N, Aboulkassim T, Ongali B, et al. Complete rescue of cerebrovascular function in aged Alzheimer's disease transgenic mice by antioxidants and pioglitazone, a peroxisome proliferator-activated receptor gamma agonist. J Neurosci 2008; 28: 9287-96.

72. Fernandez-Martos CM, Atkinson RAK, Chuah MI, et al. Combination treatment with leptin and pioglitazone in a mouse model of Alzheimer's disease. Alzheimers Dement (NY) 2016; 3: 92-106.

73. Searcy JL, Phelps JT, Pancani T, et al. Long-term pioglitazone treatment improves learning and attenuates pathological markers in a mouse model of Alzheimer's disease. J Alzheimers Dis 2012; 30: 943-61.

74. Seok $H$, Lee $M$, Shin E, et al. Low-dose pioglitazone can ameliorate learning and memory impairment in a mouse model of dementia by increasing LRP1 expression in the hippocampus. Sci Rep 2019; 9: 4414.

75. Toba J, Nikkuni M, Ishizeki $M$, et al. PPARgamma agonist pioglitazone improves cerebellar dysfunction at pre-Abeta deposition stage in APPswe/PS1dE9 Alzheimer's disease model mice. Biochem Biophys Res Commun 2016; 473: 1039-44.

76. Galea E, Feinstein DL, Lacombe P. Pioglitazone does not increase cerebral glucose utilisation in a murine model of Alzheimer's disease and decreases it in wildtype mice. Diabetologia 2006; 49: 2153-61.

77. Chin-Hsiao Tseng. Rosiglitazone has a neutral effect on the risk of dementia in type 2 diabetes patients. Aging (Albany NY) 2019; 11: 2724-34.

78. Chou PS, Ho BL, Yang YH. Effects of pioglitazone on the incidence of dementia in patients with diabetes. J Diabetes Complications 2017; 31: 1053-7.

79. Lu CH, Yang CY, Li CY, et al. Lower risk of dementia with pioglitazone, compared with other second-line treatments, in metformin-based dual therapy: a population-based longitudinal study. Diabetologia 2018; 61: 562-73.

80. Heneka MT, Fink A, Doblhammer G. Effect of pioglitazone medication on the incidence of dementia. Ann Neurol 2015; 78: 284-94.

81. Sakr HF. Effect of sitagliptin on the working memory and reference memory in type 2 diabetic Sprague-Dawley rats: possible role of adiponectin receptors 1 . J Physiol Pharmacol 2013; 64: 613-23

82. D’Amico M, Di Filippo C, Marfella R, et al. Long-term inhibition of dipeptidyl peptidase-4 in Alzheimer's prone mice. Exp Gerontol 2010; 45: 202-7. 
83. Dong Q, Teng SW, Wang Y, et al. Sitagliptin protects the cognition function of the Alzheimer's disease mice through activating glucagon-like peptide-1 and BDNF-TrkB signalings. Neurosci Lett 2019; 696: 184-90.

84. Isik AT, Soysal P, Yay A, et al. The effects of sitagliptin, a DPP-4 inhibitor, on cognitive functions in elderly diabetic patients with or without Alzheimer's disease. Diabetes Res Clin Pract 2017; 123: 192-8.

85. Kim DH, Huh JW, Jang M, et al. Sitagliptin increases tau phosphorylation in the hippocampus of rats with type 2 diabetes and in primary neuron cultures. Neurobiol Dis 2012; 46: 52-8.

86. Qin L, Chong T, Rodriguez R, et al. Glucagon-like peptide-1-mediated modulation of inflammatory pathways in the diabetic brain: relevance to Alzheimer's disease. Curr Alzheimer Res 2016; 13: 1346-55.

87. Kornelius E, Lin CL, Chang HH, et al. DPP-4 Inhibitor linagliptin attenuates Abeta-induced cytotoxicity through activation of AMPK in neuronal cells. CNS Neurosci Ther 2015; 21: 549-57.

88. Kosaraju J, Holsinger RMD, Guo L, et al. Linagliptin, a dipeptidyl peptidase-4 inhibitor, mitigates cognitive deficits and pathology in the 3xTg-AD mouse model of Alzheimer's disease. Mol Neurobiol 2017; 54: 6074-84.

89. Jain S, Sharma B. Neuroprotective effect of selective DPP-4 inhibitor in experimental vascular dementia. Physiol Behav 2015; 152: 182-93.

90. Ma QH, Jiang LF, Mao JL, et al. Vildagliptin prevents cognitive deficits and neuronal apoptosis in a rat model of Alzheimer's disease. Mol Med Rep 2018; 17: 4113-9.

91. Kosaraju J, Murthy V, Khatwal RB, et al. Vildagliptin: an anti-diabetes agent ameliorates cognitive deficits and pathology observed in streptozotocin-induced Alzhei mer's disease. J Pharm Pharmacol 2013; 65: 1773-84.

92. Kosaraju J, Gali CC, Khatwal RB, et al. Saxagliptin: a dipeptidyl peptidase-4 inhibitor ameliorates streptozotocin induced Alzheimer's disease. Neuropharmacology 2013; 72: 291-300.

93. Navarro M, Rodriquez de Fonseca F, Alvarez E, et al. Colocalization of glucagon- like peptide-1 (GLP-1) receptors, glucose transporter GLUT-2, and glucokinase mRNAs in rat hypothalamic cells: evidence for a role of GLP-1 receptor agonists as an inhibitory signal for food and water intake. J Neurochem 1996; 67: 1982-91.

94. Campos RV, Lee YC, Drucker DJ. Divergent tissue-specific and developmental expression of receptors for glucagon and glucagonlike peptide-1 in the mouse. Endocrinology 1994; 134: 2156-64.

95. Calvo JC, Yusta B, Mora F, et al. Structural characterization by affinity cross-linking of glucagon-like peptide-1(7-36)amide receptor in rat brain. J Neurochem 1995; 64: 299-306.

96. Chen S, Liu AR, An FM, et al. Amelioration of neurodegenerative changes in cellular and rat models of diabetes-related Alzheimer's disease by exendin-4. Age (Dordr) 2012; 34: 1211-24.

97. Solmaz V, Çınar BP, Yiğittürk G, et al. Exenatide reduces TNF-alpha expression and improves hippocampal neuron numbers and memory in streptozotocin treated rats. Eur J Pharmacol 2015; 765: 482-7.

98. Xu W, Yang Y, Yuan G, et al. Exendin-4, a glucagon-like peptide-1 receptor agonist, reduces Alzheimer disease-associated tau hyperphosphorylation in the hippocampus of rats with type 2 diabetes. J Investig Med 2015; 63: 267-72.

99. Li Y, Duffy KB, Ottinger MA, et al. GLP-1 receptor stimulation reduces amyloid-beta peptide accumulation and cytotoxicity in cellular and animal models of Alzheimer's disease. J Alzheimers Dis 2010; 19: 1205-19.

100. Chen S, Tang Q, Wang Y, et al. Evidence of metabolic memory-induced neurodegeneration and the therapeutic effects of glucagon-like peptide-1 receptor agonists via Forkhead box class O. Biochim Biophys Acta Mol Basis Dis 2019; 1865: 371-7.

101. Parthsarathy V, Hölscher C. Chronic treatment with the GLP1 analogue liraglutide increases cell proliferation and differentiation into neurons in an AD mouse model. PLoS One 2013; 8: e58784.

102. Yan W, Pang M, Yu Y, et al. The neuroprotection of liraglutide on diabetic cognitive deficits is associated with improved hippocampal synapses and inhibited neuronal apoptosis. Life Sci 2019; 231: 116566.

103. McClean PL, Gault VA, Harriott P, et al. Glucagon-like peptide- 1 analogues enhance synaptic plasticity in the brain: a link between diabetes and Alzheimer's disease. Eur J Pharmacol 2010; 630: 158-62.

104. Long-Smith CM, Manning S, McClean PL, et al. The diabetes drug liraglutide ameliorates aberrant insulin receptor localisation and signalling in parallel with decreasing both amyloid-beta plaque and glial pathology in a mouse model of Alzheimer's disease. Neuromolecular Med 2013; 15: 102-14.

105. Qi L, Ke L, Liu X, et al. Subcutaneous administration of liraglutide ameliorates learning and memory impairment by modulating tau hyperphosphorylation via the glycogen synthase kinase-3beta pathway in an amyloid beta protein induced alzheimer disease mouse model. Eur J Pharmacol 2016; 783: 23-32.

106. Yang Y, Zhang J, Ma D, et al. Subcutaneous administration of liraglutide ameliorates Alzheimer-associated tau hyperphosphorylation in rats with type 2 diabetes. J Alzheimers Dis 2013; 37: 637-48.

107. Batista AF, Forny-Germano L, Clarke JR, et al. The diabetes drug liraglutide reverses cognitive impairment in mice and attenuates insulin receptor and synaptic pathology in a non-human primate model of Alzheimer's disease. J Pathol 2018; 245: 85-100.

108. McClean PL, Hölscher C. Liraglutide can reverse memory impairment, synaptic loss and reduce plaque load in aged APP/PS1 mice, a model of Alzheimer's disease. Neuropharmacology 2014; 76: 57-67.

109. Hansen HH, Fabricius K, Barkholt P, et al. The GLP-1 receptor agonist liraglutide improves memory function and increases hippocampal CA1 neuronal numbers in a senescence-accelerated mouse model of Alzheimer's disease. J Alzheimers Dis 2015; 46: 877-88.

110. Hansen $\mathrm{HH}$, Fabricius K, Barkholt $\mathrm{P}$, et al. Long-term treatment with liraglutide, a glucagon-like peptide-1 (GLP-1) receptor agonist, has no effect on beta-amyloid plaque load in two transgenic APP/PS1 mouse models of Alzheimer's disease. PLoS One 2016; 11: e0158205.

111. Watson KT, Wroolie TE, Tong G, et al. Neural correlates of liraglutide effects in persons at risk for Alzheimer's disease. Behav Brain Res 2019; 356: 271-8.

112. Cai HY, Yang JT, Wang ZJ. Lixisenatide reduces amyloid plaques, neurofibrillary tangles and neuroinflammation in an APP/PS1/tau mouse model of Alzheimer's disease. Biochem Biophys Res Commun 2018; 495: 1034-40.

113. Cai HY, Hölscher C, Yue XH, et al. Lixisenatide rescues spatial memory and synaptic plasticity from amyloid beta protein-induced impairments in rats. Neuroscience $2014 ; 277: 6-13$ 
114. Hölscher C. Novel dual GLP-1/GIP receptor agonists show neuroprotective effects in Alzheimer's and Parkinson's disease models. Neuropharmacology 2018; 136: 251-9.

115. Shi L, Zhang Z, Li L, et al. A novel dual GLP-1/GIP receptor agonist alleviates cognitive decline by re-sensitizing insulin signaling in the Alzheimer icv. STZ rat model. Behav Brain Res 2017; 327: 65-74.

116. Li T, Jiao JJ, Hölscher C, et al. A novel GLP-1/GIP/Gcg triagonist reduces cognitive deficits and pathology in the $3 \times T$ Tg mouse model of Alzheimer's disease. Hippocampus 2018; 28: 358-72.

117. Tai J, Liu W, Li Y, et al. Neuroprotective effects of a triple GLP-1/GIP/glucagon receptor agonist in the APP/PS1 transgenic mouse model of Alzheimer's disease. Brain Res 2018; 1678: 64-74.

118. Rizvi SM, Shakil S, Biswas D, et al. Invokana (Canagliflozin) as a dual inhibitor of acetylcholinesterase and sodium glucose co-transporter 2: advancement in Alzheimer's disease- diabetes type 2 linkage via an enzoinformatics study. CNS Neurol Disord Drug Targets 2014; 13: 447-51.

119. Shaikh S, Rizvi SM, Shakil S, et al. Forxiga (dapagliflozin): plausible role in the treatment of diabetes-associated neurological disorders. Biotechnol Appl Biochem 2016; 63: 145-50.

120. Ahmadian G, Ju W, Liu L, et al. Tyrosine phosphorylation of GluR2 is required for insulin-stimulated AMPA receptor endocytosis and LTD. EMBO J 2004; 23: 1040-50.

121. Zhao WQ, Alkon DL. Role of insulin and insulin receptor in learning and memory. Mol Cell Endocrinol 2001; 177: 125-34.

122. Rdzak GM, Abdelghany O. Does insulin therapy for type 1 diabetes mellitus protect against Alzheimer's disease? Pharmacotherapy 2014; 34: 1317-23.

123. Semprini R, Koch G, Belli L, et al. Insulin and the future treatment of Alzheimer's disease. CNS Neurol Disord Drug Targets 2016; 15: 660-4.

124. Tsuneki $H$, Yoshida $H$, Endo K, et al. Different impacts of acylated and non-acylated long-acting insulin analogs on neural functions in vitro and in vivo. Diabetes Res Clin Pract 2017; 129: 62-72.

125. Craft S, Claxton A, Baker LD, et al. Effects of regular and long-acting insulin on cognition and Alzheimer's disease biomarkers: a pilot clinical trial. J Alzheimers Dis 2017; 57: 1325-34.

126. Claxton A, Baker LD, Hanson A, et al. Long-acting intranasal insulin detemir improves cognition for adults with mild cognitive impairment or early-stage Alzheimer's disease dementia. J Alzheimers Dis 2015; 44: 897-906. 